\title{
PROTECTIVE EFFECT OF VANILLIC ACID ON OVARIECTOMY-INDUCED OSTEOPOROSIS IN
} RATS

\author{
Yong Gui Wang ${ }^{1}$, Liang Bo Jiang ${ }^{2}$, Bo Gou ${ }^{3 *}$ \\ ${ }^{1}$ Department of Orthopaedics, Xiangyang No.1 People's Hospital affiliated to Hubei University of Medicine, \\ Xiangyang, Hubei 441000, China., ${ }^{2}$ Department of Microscopic Orthopaedics, Shiyan People's Hospital \\ affiliated to Hubei University of Medicine, Shiyan, Hubei 442000, China., ${ }^{3}$ Department of Orthopaedics, \\ Shiyan People's Hospital affiliated to Hubei University of Medicine, Shiyan, Hubei 442000, China
}

* Corresponding author E-mail: bogou113@ hotmail.com

\begin{abstract}
Background: The need for an anti-osteoporotic agent is in high demand since osteoporosis contributes to high rates of disability or impairment (high osteoporotic fracture), morbidity and mortality. Hence, the present study is designed to evaluate the protective effects of vanillic acid (VA) against bilateral ovariectomy-induced osteoporosis in female Sprague-Dawley (SD) rats.

Materials and Methods: Forty healthy female adult SD rats were separated in to four groups with sham-operated control with bilateral laprotomy (Sham; $n=10)$, bilateral overiectomy (OVX; $n=10)$ group, OVX rats were orallay administrated with $50 \mathrm{mg} / \mathrm{kg}$ b.wt of VA $(\mathrm{OVX}+50 \mathrm{VA} ; n=10)$ or $100 \mathrm{mg} / \mathrm{kg} \mathrm{b.wt} \mathrm{of} \mathrm{VA}(\mathrm{OVX}+100 \mathrm{VA} ; n=10)$ for 12 weeks (post-treatment) after 4 weeks of OVX.

Results: A significant change in the body weight gain was noted in OVX group, while treatment with VA substantially reverted to normalcy. Meanwhile, the bone mineral density and content (BMD and BMC) were substantially improved on supplementation with VA. Also, the bone turnover markers like calcium (Ca), phosphorous (P), osteocalcin (OC), alkaline phosphatase (ALP) and deoxypyridinoline (DPD) and inflammatory markers (IL-1 $\beta$, IL-6, and TNF- $\alpha$ ) levels were markedly attenuated in VA-treated rats. Moreover, the biomechanical stability was greatly ameliorated with VA administration. Both the dose of VA showed potent anti-osteoporotic activity, but VA $100 \mathrm{mg}$ showed highest protective effects as compared with $50 \mathrm{mg}$ of VA.

Conclusion: Based on the outcome, we concluded that VA 100 showed better anti-osteoporotic activity by improving $\mathrm{BMD}$ and $\mathrm{BMC}$ as well as biomechanical stability and therefore used as an alternative therapy for treating postmenopausal osteoporosis.
\end{abstract}

Keywords: Osteoporosis, Vanillic acid, Ovariectomy, Antioxidant, Inflammatory markers

\section{Introduction}

Osteoporosis is a chronic metabolic bone disorder featured by diminished bone mineral density (BMD) or mass, bone architectural deterioration with increased susceptibility to fracture (Kanis et al., 2013). Globally, almost 200 million people are affected by osteoporosis and each year the rate is increasing significantly (Law et al., 2016). The prevalence rate of osteoporosis was higher in the elderly population (senile osteoporosis), especially postmenopausal women (postmenopausal osteoporosis) due to estrogenic deficiency. (Johnell and Kanis, 2006; Liu et al., 2014). Moreover, osteoporosis contributes to enormous socio-economic burden owing to high rates of disability or impairment (high osteoporotic fracture), morbidity and even contributes to death as well (Pisani et al., 2016). Hence, WHO considered osteoporosis as a critical health issue next to cardiovascular disease (Kanis et al., 2013).

Osteoporosis is a multifactorial chronic disease with a complicated pathophysiology. However, the main pathogenesis of osteoporosis is due to altered bone remodeling (imbalance between osteoclast and osteoblast), which might be due to several factors especially oxidative stress and subsequent inflammation (Sipos et al., 2009). At present many therapeutic regimens were available for treating osteoporosis especially hormone replacement therapy (HRT), selective estrogen receptor modulator, supplementation of vitamin (Vit D) and minerals (Ca and P) as well as antiosteoporotic drugs like bisphosphonates, zoledronic acid (Qaseem et al., 2008; Lin and Lane. 2003). However, these therapies may lead to severe adverse events like stroke, breast, endometrial or ovarian cancer and atypical fractures (Ross et al., 2000; Wang et al., 2013) and hence the necessity for alternative therapy (natural) with less or without any undesirable adverse effects to treat osteoporosis are in higher demand (Zhao et al., 2011).

Vanillic acid (VA; 4-hydroxy-3-methobenzoic acid) is a phenolic compound found in high concentration in many plants especially in the roots of Angelica sinensis a Chinese herb (Circosta et al., 2006). VA is an oxidized form of Vanillin and used as a flavoring agent in food industry due to its creamy odor. VA is an intermediate compound formed during the conversion of ferulic acid to vanillin (Calixto-Campos et al., 2015). Ample amount of experimental studies has indicated that its beneficial effects on the cardiovascular, nephro and neurological system (Prince et al., 
2011; Sindhi et al., 2015; Huang et al., 2008). Chou and others (2010) hinted VA exhibit potent antioxidant activity, attributing to the presence of hydroxyl and carboxyl group. Also, VA possesses anti-inflammatory activity by effectively inhibiting proinflammatory cytokines (Kim et al., 2011). Furthermore, VA exhibit hepatoprotective (Itoh et al., 2009), gastroprotective (Kim et al., 2010), anti-microbial (Delaquis et al., 2005) and chemoprotective activities (Liang et al., 2009).

Previously, VA from Sambucus williamsii Hance (SWH) a Chinese herb has been shown to exert osteoprotective effect (estrogen analog) by stimulating the production of osteoblast on UMR 106 cell model (Xiao et al., 2014). However, the anti-osteoporotic activity of VA has not completely explored in an animal model. Moreover, VA from Angelica sinensis also been reported to exhibit estrogenic activity (Circosta et al., 2006) and hence it might be a promising candidate for treating bone related disorders like osteoporosis. Therefore, the current study was plotted to elucidate the anti-osteoporotic property of VA in OVX-rat model which mimic postmenopausal osteoporosis.

\section{Materials and Methods Chemicals}

Vanillic acid, xylene, were brought from Sigma-Aldrich (St. Louis, MO, USA). Pentobarbital sodium, diethyl ether, saline, and sodium chloride were purchased from Beijing Zhongshan Golden Bridge Biotechnology Corp. Ltd., Beijing, China. All the other reagents and chemicals used in the current study were of analytical grade.

\section{Experimental rats}

Healthy, four-month-old female Sprague-Dawley (SD) rats $(260 \pm 20 \mathrm{~g})$ were purchased from the animal center of Hubei University and maintained in animal house. All the rats were caged and maintained at $24 \pm 2{ }^{\circ} \mathrm{C}$ with free access to standard rat chow and water (ad libitum) rats were acclimated to lab condition for a week before the experiment. The current animal experiment was approved by the Hubei University ethical committee board (HUM1838-2015) that are carried out by following the Animal Welfare Act and Animal Welfare Regulations, as well as the following the Guidelines for the Care and Use of Laboratory Animals.

\section{Experimental design}

For surgical procedure, all the rats fasted overnight and anesthetized with pentobarbital sodium $(50 \mathrm{mg} / \mathrm{kg})$ injected intraperitoneally (i.p). Rats underwent either bilateral laparotomy alone (Sham group; $n=10$ ) or bilateral ovariectomy (OVX group; $n=30$ ) via midline dorsal incision. During the surgical procedure, all the rats were monitored for its vitals to avoid any mortality. After 4 weeks of recovery period all the OVX rats were divided into 3 groups as OVX alone $(n=10)$, OVX rats were orally administrated (p.o; by oral gavage) with $50 \mathrm{mg} / \mathrm{kg}$ b.wt of $\mathrm{VA} /$ day $(\mathrm{OVX}+50 \mathrm{VA} ; n=10)$ or $100 \mathrm{mg} / \mathrm{kg}$ b.wt of VA/day $(\mathrm{OVX}+100 \mathrm{VA} ; n=10)$ for 12 successive weeks. Whereas the sham-operated rats received distilled water, also VA was dissolved in distilled water.

\section{Sample collection}

For collecting $24 \mathrm{~h}$ urine sample (during fasting condition) after the experiment, all the rat was housed in an individual metallic cage, and urine samples were collected from each rat (cage) filtered using a special caged filter/separator. Then, the urine sample was acidified with $2 \mathrm{~mL}$ of HCL $(1 \mathrm{~mol} / \mathrm{L})$. All the overnight fasted rats were euthanized (on the $113^{\text {th }}$ day after 16 weeks) by diethyl ether via i. p, and the blood samples were collected from the abdominal aorta for the separation of serum (centrifuged at $3200 \mathrm{x} g$ for $20 \mathrm{~min}$ ) and stored at $-20{ }^{\circ} \mathrm{C}$ until analysis. Femurs were dissected from each rat and wrapped in saline soaked cotton gauze and stored at $-20{ }^{\circ} \mathrm{C}$ until biomechanical examination. Also, the uterus was removed, and its dry weight was measured by calculating uterus index (dry uterus wt in mg/ body wt in $\mathrm{g}$ ). The body weight was measured every week and segregated as initial, $4^{\text {th }}, 8^{\text {th }}, 12^{\text {th }}$, $16^{\text {th }}$ (final) weeks.

\section{Biochemical analysis}

The concentration of serum calcium (S-Ca), phosphorous (S-P) and alkaline phosphatase (ALP) as well as urinary calcium (U-Ca), phosphorous (U-P) and creatinine (U-Cr) were determined by using diagnostic kit from Beijing Zhongshan Golden Bridge Biotechnology Corp. Ltd., Beijing, China, using atomic absorption spectrometer (Leeman-Labs, MA, USA). Also, the levels of serum osteocalcin (S-OC) was evaluated using a rat OC ELISA kit Biovalue Biomedical Engineering Co., Ltd., Shanghai, China in accordance with manufacturer instruction. Moreover, urinary deoxypyridinoline (U-DPD) concentration was assayed by comparative enzyme immunoassay using METRATM DPA ELISA kit from Quidel Corp., CA, USA. Both urinary Ca and DPD were expressed as the ratio of Cr concentration (Ca or DPD/Cr). 


\section{Assay of inflammatory markers}

The levels of inflammatory markers (proinflammatory cytokines) like TNF- $\alpha$, IL-1 $\beta$, and IL-6 in serum samples were determined using the commercially ELISA kits provided by Thermo Fisher Scientific Inc, (MA, USA) by following the supplier protocols.

\section{Bone analysis by dual energy X-ray absorptiometry (DEXA)}

Both bone mineral content (BMC) and bone mineral density (BMD) of the femur were quantified at the end of the experiment by LUNAR PRODIGY DPXA-IQ-7040 (GE Healthcare, WI, USA) using small animal scan mode specifically for rats (Shen et al., 2010). The values were calculated by a DEXA inbuilt software package (Encore, GE Healthcare, WI, USA).

\section{Biochemical examination of Femur (3-point bending test)}

The femur was thawed (brought to room temperature) and unwrapped from gauze to examine the biomechanical stability (3-point bending test) using a testing machine -MTS 858 Mini Bionix (MTS Systems Corp., MN, USA) as instructed by manufacturer instruction. Before testing the femur length and midshaft diameter were measured by caliper. The femur diaphysis biomechanical stability was determined at a speed of $2 \mathrm{~mm} / \mathrm{min}$, and the load-deformation curve was calculated (special software) as mentioned by Zhang et al. (2012). From the loaddeformation curve the energy absorption, maximum load, stiffness, young modulus and maximum stress were obtained.

\section{Statistical analysis}

Data were reported as means \pm standard deviation (SD). Significance between each experimental group was evaluated using one-way analysis of variance (ANOVA) with the help of SPSS software version 23 (IBM Inc., IL, USA). The significance was determined by least significant difference (LSD) using a post-hoc multi-comparison test. $p$-value less than 0.05 was indicated as statistically significant.

\section{Results and Discussion}

Vanillic acid is one of the major active phytocomponents of several Chinese herbs like Angelica sinensis, Sambucus siebolddiana, Sambucus williamsii, Rhododendron dauricum. Previously, VA has been reported to exhibit estrogenic activity, and hence we hypothesize that VA may favor the anti-osteoporotic property by acting as estrogen analog (Xiao et al., 2014). Since, estrogen plays a critical role in maintaining bone mineralization and remodeling (turnover) process via regulating the balance between osteoclast and osteoblast (Ozgocmen et al., 2007). Hence, the present experiment was designed to evaluate the anti-osteoporotic property of VA in ovariectomy-induced rat model which mimic postmenopausal osteoporosis. Figure 1 epitomized the effect of VA on body weight (A), and uterus index (B) of sham-operated control and experimental rats. To confirm the removal of the uterus and lack of estrogen activity, we evaluate both body weight and uterine index (UI) in rats. Body weight was weighed every week using digital portal scale (Model 1450, Alpha Scales Ltd, Beijing, China). Throughout the experiment, the body weight was significantly higher $(\mathrm{p}<0.01)$ in OVX rats owing to lack of ovarian related hormones especially estrogen on equivalence with shamoperated control rats, despite similar food consumption. Whereas, 13-week treatment (post) with VA (50 and 100 $\mathrm{mg} / \mathrm{kg} \mathrm{b.wt)}$ considerably alleviated $(\mathrm{p}<0.01)$ those excessive body weight gain which was observed in OVX rats owing to estrogenic activity. Several studies had shown that the substance or drug with estrogenic activity could significantly lower the ovariectomy-induced weight gain (Rachon et al., 2007 and 2008). Also, the UI value was substantially lowered in OVX rats and thus proving that the surgical procedure (atrophy of uterus) was successful. While OVX rats supplemented with VA, greatly enhanced the UI value as VA can improve the uterine tissue re-growth or repair due to estrogenic property. 
Wang et al., Afr J Tradit Complement Altern Med., (2017) 14 (4): 31-38

https://doi.org/10.21010/ajtcam.v14i4.4

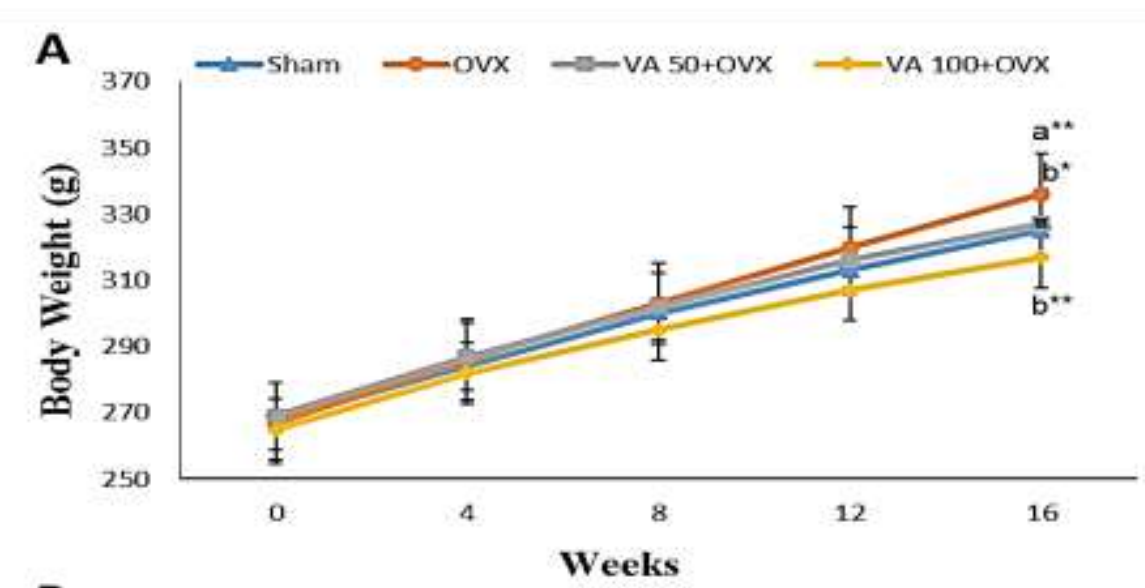

B

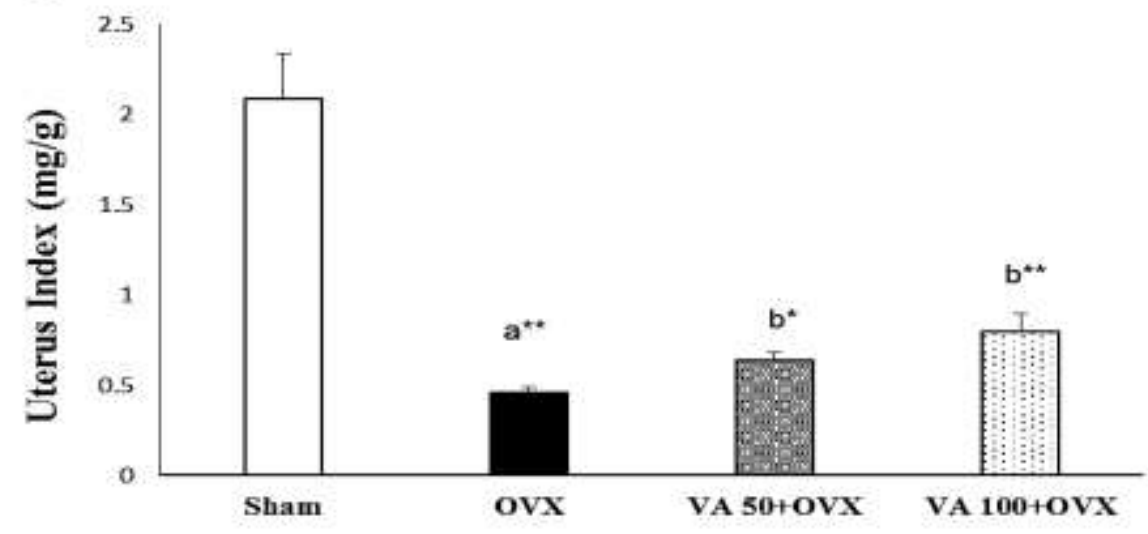

Figure 1: Effect of VA on body weight (A) and uterus index (B) of sham-operated control and experimental rats. Data were reported as means \pm S.D for 10 rats in each group. $* * p<0.01$, ${ }^{*} p<0.05$ (a) OVX group compared with the sham control group, (b) VA 50 and 100 compared with the OVX induced group.

The degree of bone mass or density loss are directly indicated by elevated bone turnover markers like ALP, OS, Ca, P and DPD in serum and urinary sample (Law et al., 2016; Elshal et al., 2013). Hence, the effect of VA on serum and urinary bone turnover markers (osteoclastic factors) of sham-operated control and experimental rats were investigated (Table 1) to evaluate the bone loss. The biochemical serum bone markers like S-ALP, S-OS and urinary bone markers like $\mathrm{U}-\mathrm{Ca} / \mathrm{Cr}, \mathrm{P} / \mathrm{Cr}$, and $\mathrm{DPD} / \mathrm{Cr}$ were concomitantly elevated in $\mathrm{OVX}$ group. However, no significant changes were noted in S-Ca or S-P in any of the group. Administration with $50(\mathrm{p}<0.05)$ or $100(\mathrm{p}<0.01) \mathrm{mg}$ of VA notably prevented the escalation of those biochemical markers (S-ALP, S-OS, U-Ca/Cr, P/Cr and DPD/Cr) in OVX rats by upregulating osteoblastic and downregulating osteoclastic related gene and thus maintain the osteogenic process. Zhang and his co-workers in 2011 indicated that SWH (rich in VA) could significantly reverse the biochemical bone markers to normal level attributing to the phenolic compounds (estrogenic activity) in OVX mice model.

The pathophysiology of osteoporosis is due to altered bone remodeling (imbalance between osteoclast and osteoblast), which might be due to several factors especially oxidative stress and subsequent inflammation (Sipos et al., 2009). Effect of VA on the concentration of serum inflammatory markers in sham-operated control and experimental rats were presented in Table.2. The concentrations of inflammatory markers like TNF- $\alpha$, IL-1 1 , and IL-6 of ovariectomized rats were exorbitantly increased $(p<0.01)$ compared with sham-operated rats. Grassi and others $(2007)$ have demonstrated that deficiency of estrogen in OVX mice can trigger free radical generation in bone marrow and subsequently initiate the inflammatory cascade by upregulating the expression of various pro-inflammatory cytokines particularly TNF- $\alpha$ through activating T cells. On comparison with OVX group, VA treated group $(50 ; p<0.05$ and $100 ; \mathrm{p}<0.01)$ markedly lowered the concentrations of those inflammatory markers. Our results are in corroboration with the outcome of Orsal and his colleagues (2013), they also inferred that the serum levels of IL-1 $\beta$, IL-6 and TNF- $\alpha$ were concomitantly increased in OVX induced rats as compared to sham-operated rats. Supplementation of vanillic acid (O-methyl group) can markedly downregulate various pro-inflammatory cytokines like TNF- $\alpha$, IL-1 $\beta$, IL-6 in an animal model (Sindhu et al., 2015). 
Wang et al., Afr J Tradit Complement Altern Med., (2017) 14 (4): $31-38$

https://doi.org/10.21010/ajtcam.v14i4.4

Table 1: Effect of VA in serum and urinary bone turnover markers (osteoclastic factors) of sham-operated control and experimental rats

\begin{tabular}{lllll}
\hline Parameters & Sham control & OVX & VA 50 + OVX & VA 100 + OVX \\
\hline S-ALP (U/L) & $112.40 \pm 10.57$ & $253.01 \pm 31.03 \mathrm{a}^{* *}$ & $183.95 \pm 22.50 \mathrm{~b}^{*}$ & $152.89 \pm 19.70 \mathrm{~b}^{* *}$ \\
$\mathbf{S - O C}(\mathbf{n m o l} / \mathbf{L})$ & $11.61 \pm 1.03$ & $17.76 \pm 1.19 \mathrm{a}^{* *}$ & $14.15 \pm 1.25 \mathrm{~b}^{*}$ & $12.98 \pm 0.54 \mathrm{~b}^{* *}$ \\
$\mathbf{S - C a}(\mathbf{m m o l} / \mathbf{L})$ & $2.52 \pm 0.22$ & $2.38 \pm 0.29$ & $2.32 \pm 0.42$ & $2.46 \pm 0.38$ \\
$\mathbf{S - P}(\mathbf{m m o l} / \mathbf{L})$ & $2.22 \pm 0.31$ & $2.08 \pm 0.18$ & $2.13 \pm 0.28$ & $2.25 \pm 0.33$ \\
$\begin{array}{l}\mathbf{U}-\mathbf{C a} / \mathbf{C r} \\
(\mathbf{m m o l} / \mathbf{m m o l})\end{array}$ & $0.28 \pm 0.02$ & $0.50 \pm 0.07 \mathrm{a}^{* *}$ & $0.39 \pm 0.04 \mathrm{~b}^{*}$ & $0.31 \pm 0.90 \mathrm{~b}^{* *}$ \\
$\begin{array}{l}\mathbf{U}-\mathbf{P} / \mathbf{C r} \\
(\mathbf{m m o l} / \mathbf{m m o l})\end{array}$ & $4.36 \pm 0.42$ & $6.05 \pm 0.68 \mathrm{a}^{* *}$ & $5.21 \pm 0.51 \mathrm{~b}^{*}$ & $4.89 \pm 0.72 \mathrm{~b}^{* *}$ \\
$\begin{array}{l}\mathbf{U}-\mathbf{D P D} / \mathbf{C r} \\
(\mathbf{m m o l} / \mathbf{m m o l})\end{array}$ & $60.21 \pm 7.11$ & $98.32 \pm 10.63 \mathrm{a}^{* *}$ & $76.05 \pm 8.82 \mathrm{~b}^{*}$ & $68.00 \pm 9.22 \mathrm{~b}^{* *}$ \\
\hline
\end{tabular}

Data were reported as means \pm S.D for 10 rats in each group. $* * p<0.01$, $* p<0.05$ (a) OVX group compared with the sham control group, (b) VA 50 and 100 compared with the OVX induced group.

Table 2: Effect of VA on the concentration of serum inflammatory markers in sham-operated control and experimental rats

\begin{tabular}{lllll}
\hline Parameters & Sham control & OVX & VA 50 + OVX & VA 100 + OVX \\
\hline TNF- $\boldsymbol{\alpha}(\mathrm{ng} / \mathrm{mL})$ & $12.45 \pm 11.38$ & $21.01 \pm 25.40 \mathrm{a}^{* *}$ & $16.83 \pm 14.92 \mathrm{~b}^{*}$ & $14.78 \pm 10.57 \mathrm{~b}^{* *}$ \\
IL-1及 $(\mathrm{pg} / \mathrm{mL})$ & $68.59 \pm 9.04$ & $97.59 \pm 12.10 \mathrm{a}^{* *}$ & $84.82 \pm 11.25 \mathrm{~b}^{*}$ & $73.92 \pm 8.83 \mathrm{~b}^{* *}$ \\
IL-6 $(\mathrm{pg} / \mathrm{mL})$ & $96.31 \pm 11.62$ & $169.11 \pm 21.23 \mathrm{a}^{* *}$ & $131.58 \pm 14.11 \mathrm{~b}^{*}$ & $110.46 \pm 18.29 \mathrm{~b}^{* *}$ \\
\hline
\end{tabular}

Data were reported as means \pm S.D for 10 rats in each group. $* * p<0.01$, $* \mathrm{p}<0.05$ (a) OVX group compared with the sham control group, (b) VA 50 and 100 compared with the OVX induced group.

Bone mineral content, bone mineral density, and bone biomechanical strength are the major factors that highlight the bone quality (mineralization and resorption) and rigidity (Rachon et al., 2007). Hence for the present study, we evaluated the bone quality and mass by assessing the femoral BMC and BMD using DEXA. Figure 2 depicts the effect of VA on the BMC and BMD in the left femur of sham-operated control and experimental rats. Both BMD and BMC levels are dramatically suppressed $(\mathrm{p}<0.01)$ in the OVX rats than sham-operated control rats. Ample amount of researcher had already proved that the bone mass-BMD and BMC (Femur/Tibia or lumbar) were substantially reduced in estrogen deficiency OVX-induced animal model (Miao et al., 2014; Devareddy et al., 2008). Treatment for 13 weeks with VA $50(\mathrm{p}<0.05)$ and $100 \mathrm{mg} / \mathrm{kg}(\mathrm{p}<0.01)$ in OVX rats considerably potentiated the levels of both BMC and BMD as compared to OVX rats. Previously, a vanillic acid rich fraction of Sambucus sieboldiana presented better inhibitory effects on bone resorption by ameliorating the levels of bone mass (BMD and BMC), as compared to other fractions of Sambucus sieboldiana in OVX mice model (Li et al., 1998). 
Wang et al., Afr J Tradit Complement Altern Med., (2017) 14 (4): 31-38

https://doi.org/10.21010/ajtcam.v14i4.4

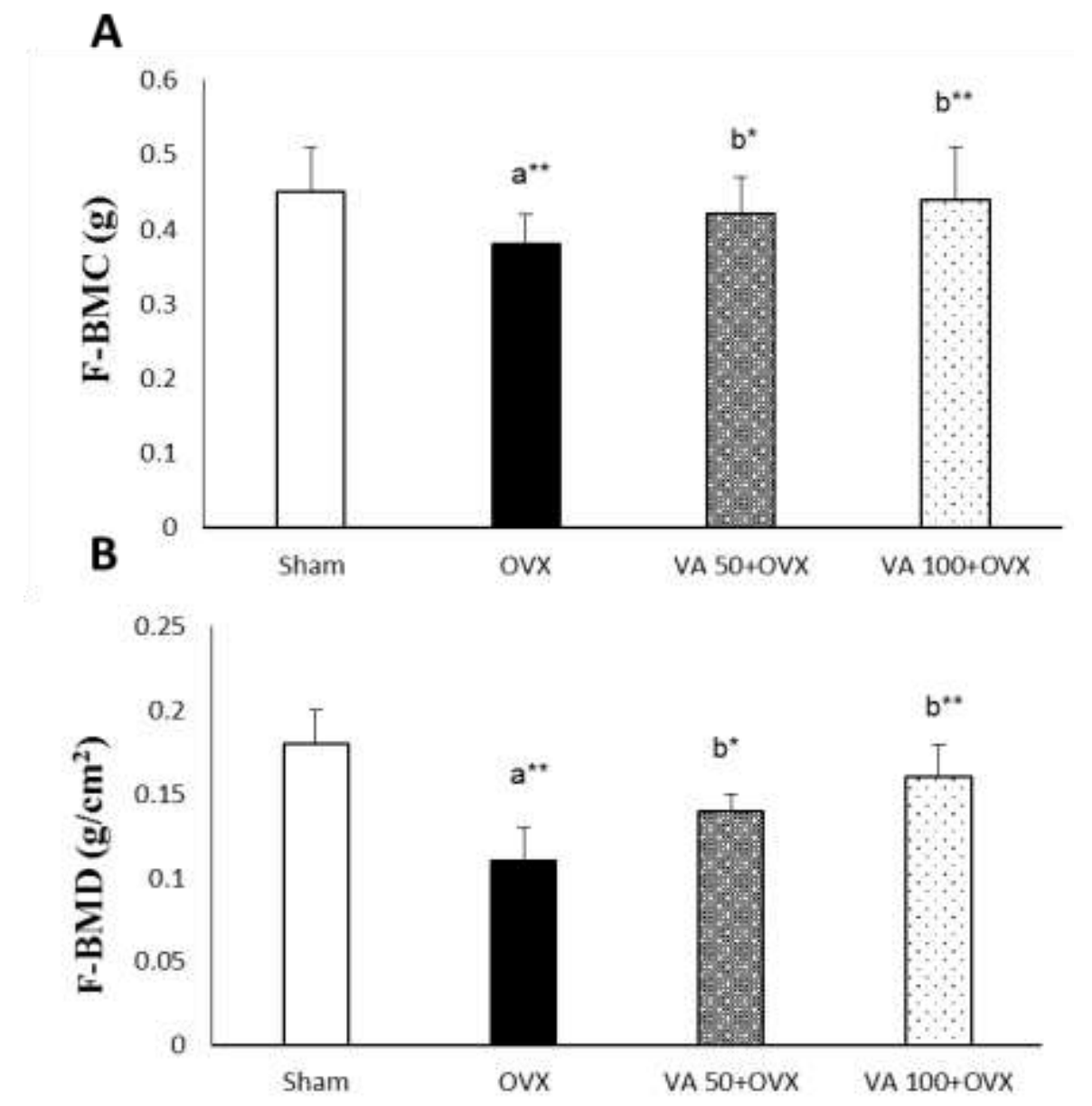

Figure 2: Effect of VA on the bone mineral content (BMC; A) and bone mineral density (BMD; B) in the femur of sham-operated control and experimental rats. Data were reported as means \pm S.D for 10 rats in each group. $* * p<0.01$, $* p<0.05$ (a) OVX group compared with the sham control group, (b) VA 50 and 100 compared with the OVX induced group.

Finally, the biomechanical competence of femur was quantified by 3 bending testing, which portraits the bone rigidity and strength, under force or stress. The biomechanical indexes of the bone are the more reliable test to evaluate the anti-osteoporotic activity of any drug (Ye et al., 2014). Effect of VA on biomechanical parameters (bending test) in left femur diaphysis of sham-operated control and experimental rats were represented in Table 3 . All the levels of energy, maximum load, stiffness, and maximum stress were drastically reduced in OVX rats due to increased inflammatory response, which triggers the elevated bone resorption and lowers bone mass. However, VA 50 and 100 consumed OVX rats considerably increased the levels of energy, maximum load, stiffness, and maximum stress, almost equivalent to Sham-operated control rats. Xiao and his colleagues (2011) had pointed out that Sambucus williamsii Hance (SWH) fraction with rich phenolic acids like Vanillic acid would account for the increased biochemical strength (bone rigidity) and bone quality, thus endorsing its osteoprotective property. Similarly, VA also acts on estrogen receptor and stimulate osteoblast synthesis and thereby maintain osteogenesis process to increase the bone mass as well as bone quality. Both the doses of VA displayed potent anti-osteoporotic activity, but VA 100 mg showed highest osteoprotective effects than $50 \mathrm{mg}$ of VA.

Table 3: Effect of VA on biomechanical parameters (bending test) in left femur diaphysis of sham-operated control and experimental rats

\begin{tabular}{lllll}
\hline Parameters & Sham control & OVX & VA 50 + OVX & VA 100+ OVX \\
\hline Energy (N) & $52.89 \pm 6.57$ & $42.01 \pm 7.03 \mathrm{a}^{* *}$ & $47.95 \pm 9.50 \mathrm{~b}^{*}$ & $51.06 \pm 9.70 \mathrm{~b}^{* *}$ \\
Maximum load (N) & $118.61 \pm 10.03$ & $97.76 \pm 11.19 \mathrm{a}^{* *}$ & $107.14 \pm 12.25 \mathrm{~b}^{*}$ & $113.80 \pm 10.54 \mathrm{~b}^{* *}$ \\
$\begin{array}{l}\text { Stiffness (N/mm) } \\
\text { Young Modulus }\end{array}$ & $176.02 \pm 20.22$ & $158.18 \pm 14.45 \mathrm{a}^{* *}$ & $170.12 \pm 19.34 \mathrm{~b}^{*}$ & $162.06 \pm 19.38 \mathrm{~b}^{* *}$ \\
$\begin{array}{l}\text { MPa) } \\
\begin{array}{l}\text { Maximum Stress } \\
(\mathbf{M P a})\end{array}\end{array}$ & $6934.22 \pm 823.31$ & $5124.82 \pm 735.18 \mathrm{a}^{* *}$ & $6002.64 \pm 834.28 \mathrm{~b}^{*}$ & $5545.25 \pm 656.33 \mathrm{~b}^{* *}$ \\
& $201.28 \pm 23.02$ & $155.50 \pm 18.07 \mathrm{a}^{* *}$ & $177.23 \pm 19.04 \mathrm{~b}^{*}$ & $190.31 \pm 22.90 \mathrm{~b}^{* *}$ \\
\hline
\end{tabular}

Data were reported as means \pm S.D for 10 rats in each group. $* * p<0.01, * p<0.05$ (a) OVX group compared with the sham control group, (b) VA 50 and 100 compared with the OVX induced group. 


\section{Wang et al., Afr J Tradit Complement Altern Med., (2017) 14 (4): $31-38$ \\ https://doi.org/10.21010/ajtcam.v14i4.4}

Some limitations of the present studies are the avoidance of other bone like tibia or vertebrae for BMD or biomechanical analysis and not the usage of positive control (estrogen) for comparison. Moreover, the microarchitecture of the femur was not analyzed, but those limitations are taken into consideration for our upcoming studies.

\section{Conclusion}

The present study clearly showed that treatment with VA 50 and 100 substantially abolish the body weight gain, bone turnover markers (Ca, P, OC, ALP, and DPD) as well as inflammatory markers (IL-1 $\beta$, IL-6, and TNF- $\alpha$ ) in OVX rats. In addition, the BMD, BMC, and biomechanical stability or strength were considerably ameliorated on supplementation with 50 and $100 \mathrm{mg}$ of VA. Both the dose of VA exhibit potent anti-osteoporotic activity, but VA 100 mg showed maximum osteoprotective than $50 \mathrm{mg}$ of VA. Therefore, VA can be recommended as an alternative therapy for treating postmenopausal osteoporosis. However, a mechanism related to its osteoporotic activity need to be explored in future studies.

\section{Acknowledgement}

Authors are thankful to the Hubei University for funding this study (HUM-34728).

\section{Declaration of conflict of interest}

The authors declare no conflicts of interest to disclose for the present experiment

\section{References}

1. Calixto-Campos, C., Carvalho, T. T., Hohmann, M. S., Pinho-Ribeiro, F. A., Fattori, V., Manchope, M. F. et al. (2015). Vanillic acid inhibits inflammatory pain by inhibiting neutrophil recruitment, oxidative stress, cytokine production, and NFאB activation in Mice. J. Nat. Prod., 78(8): 1799-1808.

2. Chou, T. H., Ding, H. Y., Hung, W. J., \& Liang, C. H. (2010). Antioxidative characteristics and inhibition of $\alpha$-melanocyte-stimulating hormone-stimulated melanogenesis of vanillin and vanillic acid from Origanum vulgare. Exp. Dermatol., 19(8): 742-750.

3. Circosta, C., Pasquale, R. D., Palumbo, D. R., Samperi, S., \& Occhiuto, F. (2006). Estrogenic activity of standardized extract of Angelica sinensis. Phytother. Res., 20(8): 665-669.

4. Delaquis, P., Stanich, K., \& Toivonen, P. (2005). Effect of pH on the inhibition of Listeria spp. by vanillin and vanillic acid. J. Food Prot., 68(7): 1472-1476.

5. Devareddy, L., Hooshmand, S., Collins, J. K., Lucas, E. A., Chai, S. C., \& Arjmandi, B. H. (2008). Blueberry prevents bone loss in ovariectomized rat model of postmenopausal osteoporosis. J. Nutr. Biochem., 19(10): 694699.

6. Elshal, M. F., Almalki, A. L., Hussein, H. K., \& Khan, J. A. (2013). Synergistic antiosteoporotic effect of Lepidium sativum and alendronate in glucocorticoid-induced osteoporosis in Wistar rats. Afr. J. Tradit. Complement. Altern. Med., 10(5): 267-273.

7. Grassi, F., Tell, G., Robbie-Ryan, M., Gao, Y., Terauchi, M., Yang, X.et al. (2007). Oxidative stress causes bone loss in estrogen-deficient mice through enhanced bone marrow dendritic cell activation. Proc. Natl. Acad. Sci., 104(38): 15087-15092.

8. Huang, S. M., Hsu, C. L., Chuang, H. C., Shih, P. H., Wu, C. H., \& Yen, G. C. (2008). Inhibitory effect of vanillic acid on methylglyoxal-mediated glycation in apoptotic Neuro-2A cells. Neurotoxicol., 29(6): 1016-1022.

9. Johnell, O., \& Kanis, J. A. (2006). An estimate of the worldwide prevalence and disability associated with osteoporotic fractures. Osteoporos. Int., 17(12): 1726-1733.

10. Kanis, J. A., McCloskey, E. V., Johansson, H., Cooper, C., Rizzoli, R., \& Reginster, J. Y. (2013). European guidance for the diagnosis and management of osteoporosis in postmenopausal women. Osteoporos. Int., 24(1): 23-57.

11. Kim, M. C., Kim, S. J., Kim, D. S., Jeon, Y. D., Park, S. J., Lee, H. S., \& Hong, S. H. (2011). Vanillic acid inhibits inflammatory mediators by suppressing NF- $\mathrm{B}$ in lipopolysaccharide stimulated mouse peritoneal macrophages. Immunopharmacol. Immunotoxicol., 33(3): 525-532.

12. Kim, S. J., Kim, M. C., Um, J. Y., \& Hong, S. H. (2010). The beneficial effect of vanillic acid on ulcerative colitis. Molecules, 15(10): 7208-7217.

13. Law, Y. Y., Chiu, H. F., Lee, H. H., Shen, Y. C., Venkatakrishnan, K., \& Wang, C. K. (2016). Consumption of onion juice modulates oxidative stress and attenuates the risk of bone disorders in middle-aged and post-menopausal healthy subjects. Food Funct., 7(2): 902-912.

14. Li, H., Li, J., Prasain, J. K., Tezuka, Y., Namba, T., Miyahara, T. et al. (1998). Antiosteoporotic Activity of the Stems of Sambucus sieboldiana. Biol. Pharma. Bull., 21(6): 594-598. 
15. Liang, J. A., Wu, S. L., Lo, H. Y., Hsiang, C. Y., \& Ho, T. Y. (2009). Vanillin inhibits matrix metalloproteinase-9 expression through down-regulation of nuclear factor- $\mathrm{kB}$ signaling pathway in human hepatocellular carcinoma cells. Mol. Pharmacol., 75(1): 151-157.

16. Lin, J. T., \& Lane, J. M. (2003). Bisphosphonates. J. Am. Acad. Orthop. Surg., 11(1): 1-4.

17. Liu, X., Zhang, R., Zhou, Y., Yang, Y., Si, H., Li, X., \& Liu, L. (2014). The Effect of Astragalus Extractive on Alveolar Bone Rebuilding Progress of Tooth Extracted Socket of Ovariectomied Rats. Afr. J. Tradit. Complement. Altern. Med., 11(5): 91-98.

18. Miao, Q., Li, J. G., Miao, S., Hu, N., Zhang, J., Zhang, S. et al. (2011). The bone-protective effect of genistein in the animal model of bilateral ovariectomy: roles of phytoestrogens and PTH/PTHR1 against post-menopausal osteoporosis. Int. J. Mol. Sci., 13(1): 56-70.

19. Orsal, E., Halici, Z., Bayir, Y., Cadirci, E., Bilen, H., Ferah, I. et al. (2013). The role of carnitine on ovariectomy and inflammation-induced osteoporosis in rats. Exp. Biol. Med., 238: 1406-12.

20. Ozgocmen, S., Kaya, H., Fadillioglu, E., Aydogan, R., \& Yilmaz, Z. (2007). Role of antioxidant systems, lipid peroxidation, and nitric oxide in postmenopausal osteoporosis. Mol. Cell. Biochem., 295: 45-52.

21. Pisani, P., Renna, M. D., Conversano, F., Casciaro, E., Di Paola, M., Quarta, E., Muratore, M., \& Casciaro, S. (2016). Major osteoporotic fragility fractures: Risk factor updates and societal impact. World J. Orthoped., 7(3): 171-76.

22. Prince, P. S. M., Dhanasekar, K., \& Rajakumar, S. (2011). Preventive effects of vanillic acid on lipids, bax, bcl-2 and myocardial infarct size on isoproterenol-induced myocardial infarcted rats: a biochemical and in vitro study. Cardiovasc. Toxicol., 11(1): 58-66.

23. Qaseem, A., Snow, V., Shekelle, P., Hopkins, R., Forciea, M. A., \& Owens, D. K. (2008). Pharmacologic treatment of low bone density or osteoporosis to prevent fractures: a clinical practice guideline from the American College of Physicians. Ann. Intern. Med., 149(6): 404-415.

24. Rachon, D., Vortherms, T., Seidlovä-Wuttke, D., \& Wuttke, W. (2007). Effects of dietary equol on body weight gain, intra-abdominal fat accumulation, plasma lipids, and glucose tolerance in ovariectomized Sprague-Dawley rats. Menopause, 14(5): 925-932.

25. Rachoń, D., Vortherms, T., Seidlová-Wuttke, D., \& Wuttke, W. (2008). Effects of black cohosh extract on body weight gain, intra-abdominal fat accumulation, plasma lipids and glucose tolerance in ovariectomized SpragueDawley rats. Maturitas, 60(3): 209-215.

26. Ross, R. K., Paganini-Hill, A., Wan, P. C., \& Pike, M. C. (2000). Effect of hormone replacement therapy on breast cancer risk: estrogen versus estrogen plus progestin. J. Natl. Cancer Inst., 92(4): 328-332.

27. Shen, Y., Li, Y. Q., Li, S. P., Ma, L., Ding, L. J., \& Ji, H. (2010). Alleviation of ovariectomy-induced osteoporosis in rats by Panax notoginseng saponins. J. Nat. Med., 64(3): 336-345.

28. Sindhu, G., Nishanthi, E., \& Sharmila, R. (2015). Nephroprotective effect of vanillic acid against cisplatin induced nephrotoxicity in wistar rats: a biochemical and molecular study. Environ. Toxicol. Pharmacol., 39(1): 392-404.

29. Sipos, W., Pietschmann, P., Rauner, M., Kerschan-Schindl, K., \& Patsch, J. (2009). Pathophysiology of osteoporosis. Wien. Med. Wochenschr., 159(9-10): 230-234.

30. Wang, Z. Q., Li, J. L., Sun, Y. L., Yao, M., Gao, J., Yang, Z.et al. (2013). Chinese herbal medicine for osteoporosis: a systematic review of randomized controlled trails. Evid. Based Complement. Altern. Med., 2013.

31. Xiao, H. H., Gao, Q. G., Zhang, Y., Wong, K. C., Dai, Y., Yao, X. S., \& Wong, M. S. (2014). Vanillic acid exerts oestrogen-like activities in osteoblast-like UMR 106 cells through MAP kinase (MEK/ERK)-mediated ER signaling pathway. The J. Ster. Biochem. Mol. Biol., 144: 382-391.

32. Xiao, H. H., Dai, Y., Wan, H. Y., Wong, M. S., \& Yao, X. S. (2011). Bone-protective effects of bioactive fractions and ingredients in Sambucus williamsii HANCE. Br. J. Nutr., 106(12): 1802-1809.

33. Ye, L. I., Shan-Shan, L. Ü., Gui-Ying, T. A. N. G., Min, H. O. U., Qing, T. A. N. G., ZHANG, X. N. et al. (2014). Effect of Morinda officinalis capsule on osteoporosis in ovariectomized rats. Chin. J. Nat. Med., 12(3), $204-212$.

34. Zhang, R., Hu, S. J., Li, C., Zhang, F., Gan, H. Q., \& Mei, Q. B. (2012). Achyranthes bidentata root extract prevent OVX-induced osteoporosis in rats. J. Ethnopharmacol., 139(1): 12-18.

35. Zhang, Y., Li, Q., Wan, H. Y., Xiao, H. H., Lai, W. P., Yao, X. S., \& Wong, M. S. (2011). Study of the mechanisms by which Sambucus williamsii HANCE extract exert protective effects against ovariectomy-induced osteoporosis in vivo. Osteoporos. Int., 22(2): 703-709.

36. Zhao, X., Wu, Z. X., Zhang, Y., Yan, Y. B., He, Q., Cao, P. C., \& Lei, W. (2011). Anti-osteoporosis activity of Cibotium barometz extracts on ovariectomy-induced bone loss in rats. J. Ethnopharmacol., 137(3): 1083-1088. 